\title{
Preface: strategies to achieve long-term success of lung transplantation
}

Lung transplantation is now an established therapy used in patients suffering from respiratory failure. More than 4,000 lung transplants are reportedly performed every year worldwide (1), but achieving long-term success remains a major obstacle in the field. According to the registry data of the International Society for Heart and Lung Transplantation the median survival after lung transplantation increased from 4.7 years to 6.7 years in the last two decades (1). Although patient survival has improved over the years, most of the difference in survival is derived from the early post-transplant period. Long-term survival curves in different eras are almost parallel (1). This suggests that there have not been substantial improvements in long-term patient management, although there are many new concepts emerging in the field.

To produce this series of Annals of Translational Medicine titled "Strategies to achieve long-term success of lung transplantation", world experts in the field have generated comprehensive review articles in each specific area. The long-term success of lung transplantation depends primarily on appropriate patient selection, and Ainge-Allen and Granville have summarized all the important aspects of recipient selection in lung transplantation. Immunosuppression strategies play critical roles in protecting transplanted lungs from alloimmune responses after lung transplantation, and Chung and Dilling have contributed an updated appraisal of immunosuppression strategies. Surveillance and treatment of acute cellular rejection are critical components of maintenance, and this is discussed by Greer. Bery and Hachem provide insight into the role of antibody-mediated rejection, which has garnered increased attention recently, and related observations pertaining to immunosuppression increasing the risk of infection are also considered. Prophylaxis and management of bacterial, mycobacterial, and fungal infection in the context of lung transplantation are reviewed by various experts. Increased malignancy and its management are important aspects of post-lung transplantation in patients who subsequently undergo life-long immunosuppression, and this issue is reviewed by Shtraichman and Ahya. Importantly, alloantigen-independent injuries such as primary graft dysfunction and aspiration as well as infection activate innate immunity, which in turn provokes adaptive immune responses. Kawashima and Juvet have summarized the role of innate immunity after lung transplantation. Last but not least, chronic lung allograft dysfunction (CLAD) is a common final pathway of graft failure in the long term. Kotecha et al. have contributed an updated review of this important issue. Sato has summarized hypotheses that explain different phenotypes of CLAD, bronchiolitis obliterans syndrome, and restrictive allograft syndrome. We also included an original technical article on orthotopic rat lung transplantation, emphasizing the importance of basic research in the field. As guest editors of this series, we felt it was a tremendous opportunity to revisit the multiple complex issues surrounding lung transplantation via a collection of review articles undertaken by world experts.

In general, lung transplant recipients undergo more intensive immunosuppression than patents who receive other organs. This is because transplanted lung allografts are more vulnerable to various types of immune attack associated with innate and adaptive immunity. Notably however, intensive immunosuppression corresponds with increased susceptibility to various types of infection and malignancy. Lastly, multifactorial damage to allografted lungs ultimately drives their functional and mechanical deterioration, in the phenomenon now known as CLAD. Lung transplant recipients are destined to walk a tightrope with respect to the inevitable subtleties involved in balancing the risk of rejection with the risk of infection and other immunosuppression-related complications (Figure 1A). An important consideration in this regard is that alloimmune responses and infections or other alloantigen-independent graft injuries are not truly counter-directed, but rather their vector addition is directed toward graft failure or CLAD (Figure 1B).

True immunological tolerance has not yet been achieved in lung transplantation, and there is no "ace in the hole" for achieving long-term success in the field. Current best practice incorporates meticulous control of details, and if there is an emerging source of potential graft dysfunction, counteracting it while its magnitude remains small. This series of Annals of Translational Medicine is intended to cover the diverse array of potential sources of long-term lung allograft dysfunction and/or death. It is also aimed at encouraging lung transplant physicians to react promptly to ominous signs of events associated with lung allograft failure. Meticulous attitudes to patient care minimize the magnitude of such events, helping the graft to remain stable or "silent" in the long term. We believe such proactive patient management to maintain a silent graft is the ultimate key 

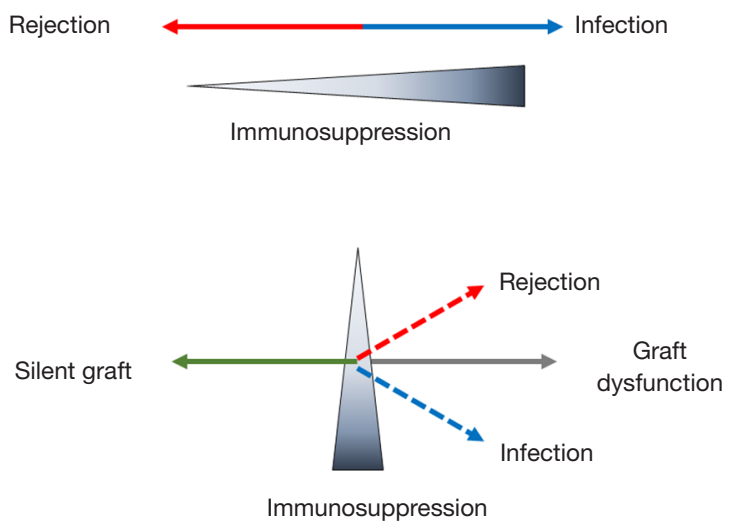

Figure 1 Conventional and new paradigms of lung allograft stability. (A) A depiction of the conventional paradigm involving the inverse relationships between immunosuppression and graft rejection and infection; (B) a new paradigm in which both rejection and infection direct the graft toward failure, but meticulous management by lung transplant physicians may counteract it.

to achieving long-term success of lung transplantation.

\section{Acknowledgments}

We thank all the authors who contributed to this series of Annals of Translational Medicine, for providing such excellent overviews of complex issues surrounding lung transplantation. We also thank Dr Owen Proudfoot from Liwen Bianji, Edanz Editing China (www.liwenbianji.cn/ac) for editing the English text of a draft of this manuscript.

Funding: This study was funded by grant JSPS KAKENHI 17H01581 (to M Sato).

\section{Footnote}

Provenance and Peer Review: This article was commissioned by the editorial office, Annals of Translational Medicine for the series "Strategies to Achieve Long-Term Success of Lung Transplantation". The article did not undergo external peer review.

Conflicts of Interest: The series "Strategies to Achieve Long-Term Success of Lung Transplantation" was commissioned by the editorial office without any funding or sponsorship. MS and DT served as the unpaid Guest Editors of the series. DT serves as an unpaid Section Editor of Annals of Translational Medicine from Oct 2019 - Sep 2020.

Ethical Statement: The authors are accountable for all aspects of the work in ensuring that questions related to the accuracy or integrity of any part of the work are appropriately investigated and resolved.

Open Access Statement: This is an Open Access article distributed in accordance with the Creative Commons AttributionNonCommercial-NoDerivs 4.0 International License (CC BY-NC-ND 4.0), which permits the non-commercial replication and distribution of the article with the strict proviso that no changes or edits are made and the original work is properly cited (including links to both the formal publication through the relevant DOI and the license). See: https://creativecommons.org/ licenses/by-nc-nd/4.0/. 


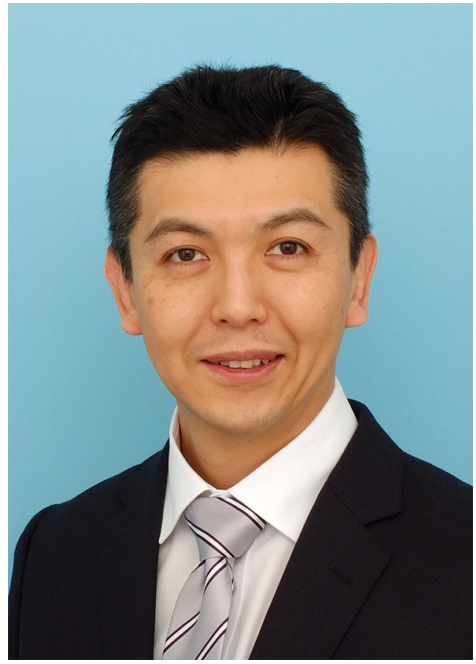

Masaaki Sato

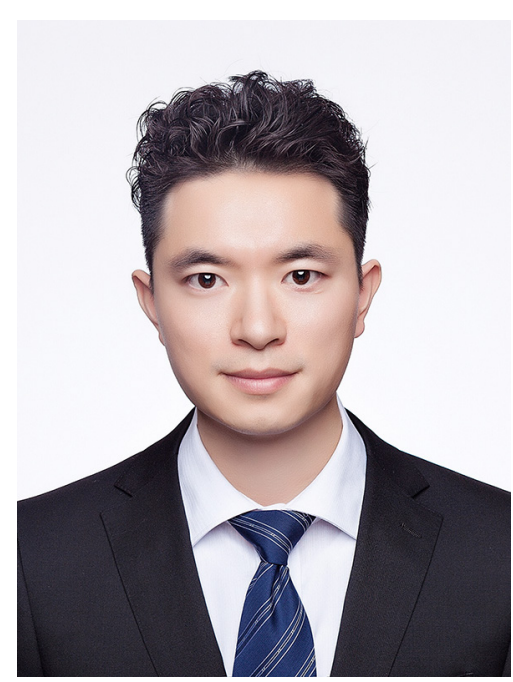

Dong Tian

\section{References}

1. Chambers DC, Cherikh WS, Harhay MO, et al. The International Thoracic Organ Transplant Registry of the International Society for Heart and Lung Transplantation: Thirty-sixth adult lung and heart-lung transplantation Report-2019; Focus theme: Donor and recipient size match. J Heart Lung Transplant 2019;38:1042-55.

Masaaki Sato ${ }^{1}$, Dong $\operatorname{Tian}^{1,2,3}$

${ }^{1}$ Department of Thoracic Surgery, University of Tokyo Graduate School of Medicine, Tokyo, Japan; ${ }^{2}$ Department of Thoracic Surgery, Affiliated Hospital of North Sichuan Medical College, Nanchong 637000, China; ${ }^{3}$ Department of Thoracic Surgery, West China Hospital, Sichuan University, Chengdu 610041, China. (Email:satom-sur@h.u-tokyo.ac.jp; 22tiandong@163.com) Submitted Jan 26, 2020. Accepted for publication Feb 13, 2020. doi: $10.21037 / \mathrm{atm} .2020 .02 .160$ View this article at: http://dx.doi.org/10.21037/atm.2020.02.160

Cite this article as: Sato M, Tian D. Preface: strategies to achieve long-term success of lung transplantation. Ann Transl Med 2020;8(6):406. doi: 10.21037/atm.2020.02.160 